\title{
Bovine Allergens in a Ruminant Clinic and Dairy Barns: Exposure Levels, Determinants, and Variability
}

\author{
Sadegh Samadi ${ }^{1,2 *}$, Dick J.J. Heederik ${ }^{2}$, Eva Zahradnik ${ }^{3}$, \\ Nancy N.J. Rietbroek ${ }^{4}$, Frank van Eerdenburg ${ }^{4}$, Ingrid Sander ${ }^{3}$, \\ Monika Raulf ${ }^{3}$ and Inge M. Wouters ${ }^{2}$
}

'Department of Occupational Health, Health Faculty, Arak University of Medical Sciences, Arak, Iran; 2Division of Environmental Epidemiology, Institute for Risk Assessment Sciences (IRAS), Utrecht University, P.O. Box 80178, 3508 TD Utrecht, The Netherlands; 3 Institute for Prevention and Occupational Medicine of the German Social Accident Insurance (IPA), Institute of the Ruhr-Universität Bochum, Bochum, Germany; ${ }^{4}$ Department of Farm Animal Health, Faculty of Veterinary Medicine, Utrecht University, P.0. Box 80178, 3584 CN Utrecht, The Netherlands

*Author to whom correspondence should be addressed. Tel: +31-30-253-9480; fax +31-30-253-9499; e-mail: samsad69@gmail.com

Submitted 30 August 2017; revised 23 February 2018; editorial decision 27 March 2018; revised version accepted 10 April 2018.

\begin{abstract}
Background: Dairy farmers may develop specific sensitization and allergic airway diseases due to bovine allergens. However, dose-response relationships are lacking, and as yet little is known on bovine allergen exposure levels.

Objective: To investigate bovine allergen exposure levels in a ruminant clinic and dairy barns, and to assess exposure determinants and variability of exposure.

Methods: Samples were collected using active and passive airborne dust measurements in a ruminant clinic and several dairy barns. Bovine allergen levels were determined by sandwich enzymelinked immunosorbent assay. Linear mixed models were applied to explore the association between bovine allergen exposure levels and potential exposure determinants. Day-to-day within-worker and between-worker exposure variability was determined, as well as how exposure determinants affect exposure variability.

Results: Bovine allergens were measureable in all samples. Personal bovine allergen exposure levels in the ruminant clinic ranged from 0.10 to $24.8 \mu \mathrm{g} / \mathrm{m}^{3}$, geometric mean (GM) $1.34 \mu \mathrm{g} / \mathrm{m}^{3}$. Exposure levels varied dependent on job titles. Personal exposure levels in dairy barns ranged from 0.10 to $46.8 \mu \mathrm{g} / \mathrm{m}^{3}, \mathrm{GM} 1.47 \mu \mathrm{g} / \mathrm{m}^{3}$. Type of bedding materials in the barns appeared to be a significant determinant of bovine allergen levels. Compost bedding, particularly, increased allergen levels. Milking by robot was the most important determinant explaining between-worker exposure variability, while
\end{abstract}


bedding was important as well. Bovine allergen levels in stationary measurements were somewhat lower than personal measurements (GM ratio 0.47). Bovine allergens could be readily detected in electrostatic dust-fall collector measurements.

Conclusion: This study provides insight in bovine allergen exposure levels and their determinants, which is a first step to investigate dose-response relationships between sensitization/allergy associated with exposure to bovine allergen levels in future studies.

Keywords: allergy; bovine allergens; dairy barn; determinant; exposure; ruminant clinic; sensitization; variability

\section{Introduction}

Workers in agricultural environment settings dealing with animals may be exposed to a variety of proteins originating from animals and plants (Samadi et al., 2013). Proteins derived from animals are classified as high-molecular-weight components, and are considered to be potent allergens (Samadi et al., 2013). Laboratory animal-derived allergens (for rat the main allergen is Rat $\mathrm{n} 1$ and for mouse, Mus n 1) were found in high concentrations in samples of airborne dust at workplaces, and thus sensitization to laboratory animal allergens and subsequent allergic airway diseases are well-known occupational disorders due to animal-derived allergens (Seward, 1999). Farm animals (e.g. cow and horse) are other well-known allergen producers (Chapman and Wood, 2001). Exposure to farm animals, particularly cow as the farm animal most commonly used, takes place in certain jobs within animal settings such as dairy farmers and veterinarians. Several different proteins have been identified as bovine antigens, but only some of them have been characterized as bovine allergens that can be found in cow hair and dander (Prahl et al., 1978). The predominant bovine allergen is Bos $\mathrm{d} 2$ (Bos domesticus 2) (Rautiainen et al., 1997), which belongs to a protein family termed lipocalins (Mantyjarvi et al., 1996). Bos d 2 can be found mainly in hair and dander.

Occupational dermatitis related to bovine dander was first described in 1948 (Epstein, 1948). Since then, several other authors published prevalence of bovine allergen sensitization as well as allergic airway diseases as a consequence of bovine allergen exposure (Terho et al., 1985; Rautalahti et al., 1987; Virtanen et al., 1988b; Ylonen et al., 1992; Doekes, 2000; Spiewak, 2001; Heutelbeck et al., 2007; Samadi et al., 2012a). A study among Finish dairy farmers by Rautalahti et al. (1987) revealed that $14 \%$ of farmers were sensitized to bovine allergen, as observed likewise in later Finnish studies (Terho et al., 1985; Virtanen et al., 1988b; Ylonen et al., 1992). This finding has been followed by a further study showing the importance of bovine-derived proteins as the primarily causative agent for development of occupational asthma, accounting for 609 out of 2609 occupational asthma cases between 1989 and 1995 (Karjalainen et al., 2000). In contrast, no associations with onset of allergic respiratory symptoms or diseases and bovine allergen sensitization were observed among Dutch, Danish, and Polish dairy farmers (Doekes, 2000; Spiewak, 2001), and a recent study reported cow sensitization to be uncommon in dairy farmers in Denmark (Schlunssen et al., 2015). Although there is no clear answer to the large discrepancies between studies, healthy worker selection bias may play a role and differences in exposure levels of obvious allergens may be part of the explanation as well.

Few studies investigated levels of bovine allergen exposures in agricultural environment settings (Virtanen et al., 1988a; Ylonen et al., 1994; Williams et al., 2011; Zahradnik et al., 2011; Schlunssen et al., 2015; Bohlandt et al., 2016; Williams et al., 2016). A study based on personal and stationary exposure measurements in 18 Finish cowsheds revealed that bovine epithelial antigen in total dust fraction was detectable with a mean level of $0.46 \mu \mathrm{g} / \mathrm{m}^{3}$ in personal measurements and a mean between 0.35 and $0.73 \mu \mathrm{g} / \mathrm{m}^{3}$ in stationary measurements (Virtanen et al., 1988a). Three recent studies using electrostatic dust-fall collectors (EDCs) for settled airborne dust demonstrated that bovine allergens were measurable in dairy farms, with substantially higher exposure levels in cow stables than within dairy farmers' dwellings (Zahradnik et al., 2011; Schlunssen et al., 2015; Bohlandt et al., 2016). All these studies prove the presence of bovine allergens in dairy barns, but still little is known on personal bovine allergen exposure levels of individuals occupationally involved within different settings in cow facilities. Therefore, the objective of this study was to investigate exposure levels of bovine allergens among veterinary medicine students and workers involved in a ruminant clinic and dairy barns. Exposure determinants and variability of exposure levels were evaluated.

\section{Materials and methods}

\section{Exposure measurements}

Exposure measurements were carried out at a ruminant clinic and 23 dairy barns as described previously in detail (Samadi et al., 2011, 2012b). Briefly, the ruminant 
clinic is sited at the Faculty of Veterinary Medicine of Utrecht University, the Netherlands. This clinic is subservient to education and research. It is a modern building comprising of cow stables, cow examination rooms and a canteen. Regular dairy barns included in the study were located near the city of Utrecht, and consisted of a confined building with freestalls. The floor of each barn consisted of a concrete slates. The freestalls were bedded with four different beddings: compost, sawdust, rubber filled mattress, and rubber mats. On the mattress and mats, an extra top layer of bedding material was also applied such as chopped straw or sawdust with the purpose of keeping cows clean and dry.

Personal inhalable dust was collected repeatedly over time for individuals dependent on job title, work-shift, and location. Repeated stationary sampling took place at different locations as well. A portable pump operating at a flow rate of $3.5 \mathrm{l} / \mathrm{min}$ was applied for personal and stationary inhalable dust measurements, using a plastic conical inhalable sampler equipped with glass fiber filters (Whatman International Ltd, Maidstone, UK). In dairy barns, the measurements were performed throughout a full work-shift, in most cases from 6:00 a.m. until 2:00 pm. Measurements for workers in the ruminant clinic were carried out during three different full workshifts: daytime, 8:00-16:00; morning, 6:00-14:00; and afternoon, 14:00-22:00. Students of veterinary medicine are categorized based on their study phase in preclinical students (1-4th years), clinical students performing uniform internships (5th year), and clinical students performing differential internships (6th year). For students, measurements were conducted throughout practical teaching work. The lower limit of detections (LODs) for personal and stationary dust measurements ranged from 0.12 to $0.13 \mathrm{mg}$ per filter. Also, 10.1 and $13.9 \%$ of personal and stationary inhalable dust measurements were, respectively, below these LODs.

EDCs collecting settled airborne dust were only applied within the ruminant clinic due to logistic constraints (Noss et al., 2008). Each EDC sampler consisted of two electrostatic cloths, with an exposed area of $0.0209 \mathrm{~m}^{2}$ for each cloth, of which one was used for allergen analysis. The EDCs were placed in different areas within the clinic at roughly $1.7 \mathrm{~m}$ above the ground level and left exposed for 14 days.

\section{Extraction and detection of bovine allergens}

Sequential extraction has been applied as previously described elsewhere (Schram et al., 2005), of which the supernatant of the second extraction step was used for bovine allergen analysis. Briefly, each personal or stationary dust sample was first eluted in $5 \mathrm{ml}$ pyrogen-free water containing $0.05 \%$ Tween-20 (PBST). EDC-settled dust samples were extracted in $20 \mathrm{ml}$. Samples were shaken for $1 \mathrm{hr}$ in an end-over-end roller at room temperature, following centrifugation for $15 \mathrm{~min}$ at $1000 \mathrm{~g}$. $10 \%$ of supernatant was harvested for endotoxin analyses. This amount was replenished with 10 times concentrated phosphate buffered saline as a second extraction step. Samples were again shaken for $1 \mathrm{hr}$ in an end-overend roller at room temperature, following centrifugation for $15 \mathrm{~min}$ at $2000 \mathrm{~g}$. Ten percent of supernatant was stored in aliquots at $-20^{\circ} \mathrm{C}$ until allergen analysis.

The levels of bovine allergens were determined through a sensitive sandwich enzyme-linked immunosorbent assay as described by Zahradnik et al. (Zahradnik et al., 2011). In brief, microtiter plates (Thermo Fisher Scientific, Roskilde, Denmark) were coated over night with $100 \mu \mathrm{l} /$ well anti-cow hair polyclonal antibodies (pAbs) and blocked for $2 \mathrm{hr}$ with $200 \mu \mathrm{l} /$ well of PBST with $1 \%$ gelatin. Afterwards, the plates were incubated for $1 \mathrm{hr}$ at $22^{\circ} \mathrm{C}$ with standards, assay controls and samples diluted in PBST. A standard curve was produced using a commercial cow dander extract (Allergon, Angelholm, Sweden) over the concentrations range $0.08-10 \mathrm{ng} / \mathrm{ml}$. Cow shed settled dust and wheat flour extract were used, respectively, as positive and negative controls. The plates were incubated for $1 \mathrm{hr}$ at $22^{\circ} \mathrm{C}$ with biotinylated anti-cow hair polyclonal antibody, followed by streptavidin-peroxidase conjugate (PolyHRP80-SA, Fitzgerald, Concord, MA, USA) and finally the plates were developed with ABTS substrate (SigmaAldrich, Steinheim, Germany). The optical density (OD) was read at $414 \mathrm{~nm}$. Sample concentrations were interpolated from a four-parameter fitted standard curve using Softmax Pro 4.7.1 (Molecular Devices, Sunnyvale, CA, USA). The lower limit of detection (LOD) was the concentration corresponding to the minimal value of the four-parameter curve fit function plus the 6-fold standard deviation of the zero standard. Each sample was analyzed using three serial dilutions in PBST (1:50, 1:100 and 1:200), and the mean of diluted concentrations was utilized as the final concentration. Bovine allergen levels were expressed as $\mu \mathrm{g} / \mathrm{m}^{3}$ or $\mu \mathrm{g} / \mathrm{mg}$ of dust for personal and stationary samples, and $\mu \mathrm{g} / \mathrm{m}^{2}$ for EDC-settled dust samples. The average LOD of the allergen assay was $0.118 \mathrm{ng} / \mathrm{ml}$. All field blank samples had bovine allergen levels below this LOD. A total of 336 samples were analyzed. The bovine allergen levels of all dust measurements exceeded the LOD of the allergen assay.

\section{Statistical analysis}

Statistical analyses were carried out applying statistical software SAS, version 9.4 (SAS Institute, Cary, NC, USA). 
The values of dust measurements below the LODs were replaced using imputation and maximum likelihood estimation (Lubin et al., 2004). This method supposes that the probability distribution of values below the LOD pursue the same as observed data and substitute each non-detectable value with a random value between zero and the estimated LOD. Relationships between dust and allergen levels were explored using Pearson correlation coefficients. Linear mixed models were fitted using the log-transformed bovine allergen levels. A forward stepwise modeling procedure was used to explore the influential exposure determinants. Variables were held in the final model when they were significantly associated with exposure $(P<0.05)$. 'Worker' was considered a random effect, while 'type of bedding materials' and other determinants were assumed as fixed effects. Exposure variability was modeled as personal (between-worker variability $\left[{ }_{\mathrm{bw}} \sigma^{2}\right]$ in average levels) and temporal (withinworker variability $\left[_{\mathrm{ww}} \sigma^{2}\right]$ from day-to-day) variability. The estimation method applied was the restricted maximum likelihood method.

\section{Results}

\section{Exposure levels}

In this study we were able to detect levels of bovine allergens in all personal and stationary measurements even with non-detectable levels of dust on some filters. The distribution of personal exposure measurements for both inhalable dust and bovine allergens stratified by job title and type of bedding are shown in Table 1. Overall, personal exposure levels to bovine allergens ranged from 0.10 to $46.8 \mu \mathrm{g} / \mathrm{m}^{3}$, with a GM of $1.39 \mu \mathrm{g} / \mathrm{m}^{3}$. Significant differences in bovine allergen levels were observed between job titles for students $(P<0.0001)$, while this was not significant for inhalable dust. The highest bovine allergens levels appeared to be among preclinical students with a GM of $3.16 \mu \mathrm{g} / \mathrm{m}^{3}$ (range $0.90-24.8 \mu \mathrm{g} / \mathrm{m}^{3}$ ), which was roughly five times higher than the GM observed for uniform students $\left(0.65 \mu \mathrm{g} / \mathrm{m}^{3}\right)$. The levels of bovine allergens for workers within the ruminant clinic were the highest during the afternoon shift (GM $\left.1.85 \mu \mathrm{g} / \mathrm{m}^{3}\right)$, roughly double the exposure during the morning (GM $0.93 \mu \mathrm{g} / \mathrm{m}^{3}$ ) and daytime (GM $1.14 \mu \mathrm{g} / \mathrm{m}^{3}$ ) shifts, but these differences were not statistically significant $(P>0.05)$. Significant differences in personal bovine allergen levels $(P<0.05$, Table 1$)$ were observed between barns using different bedding types. The highest levels to bovine allergens (GM $2.95 \mu \mathrm{g} / \mathrm{m}^{3}$ ) were observed in barns using compost bedding and these levels were significantly higher than the levels in barns with sawdust bedding ( $\mathrm{GM}$ ratio $4.8, P<0.05$ ), while other post hoc comparisons based on bedding types were not significant $(P>0.05)$. The estimated bovine allergen content levels expressed as $\mu \mathrm{g} / \mathrm{mg}$ of dust ranged from 0.05 to 20.3 , with a GM of $1.96 \mu \mathrm{g} / \mathrm{m}$. The pattern of allergen content $(\mu \mathrm{g} / \mathrm{mg})$ levels over job title, location and work-shift were different than the pattern for allergen concentration $\left(\mu \mathrm{g} / \mathrm{m}^{3}\right)$, suggesting that dust formation may partly drive the observed higher levels with certain determinants.

Stationary levels of bovine allergens ranged from 0.28 to $35.6 \mu \mathrm{g} / \mathrm{m}^{3}$, with a GM of $0.66 \mu \mathrm{g} / \mathrm{m}^{3}$ (Table 2). Overall, bovine allergen levels of stationary measurements were somewhat lower than levels in personal measurements (GM ratio 0.47). The GM bovine allergen level in cow stables within the ruminant clinic was twice as high as in examination rooms (GM ratio 2.10, $P<0.05)$. Interestingly, the overall bovine allergen level in dairy barns was four times as high as the level in the cow stable within the ruminant clinic $(P<0.05)$.

The results of bovine allergen levels in airborne-settled dust samples are presented in Table 3. Bovine allergen levels in cow barns within the ruminant clinic were significantly higher than in examination rooms (GM ratio 3.70). Bovine allergen exposure in the canteen was detectable, but far lower than in examination rooms (GM ratio 0.10).

\section{Comparisons between inhalable dust and bovine allergens}

Moderate to weak, but significant, Pearson correlations were observed between personal inhalable dust levels and bovine allergen levels ([ruminant clinic: students, $r=0.50, P<0.0001$; workers, $r=0.37, P=0.03$ ] and [dairy barns: workers, $r=0.64, P<0.0001]$ ). Correlations for stationary measurements were in the same range (ruminant clinic: $r=0.48, P=0.002$; and dairy barns: $r=0.60, P<0.0001)$.

\section{Determinants and variability of exposure levels}

Results of the mixed-effect models for personal and stationary bovine allergen levels in different barns in relation to potential exposure determinants are presented in Table 4. Looking at mixed-effect models for personal samples, compost appeared to be the strongest significant determinant of personal exposure to bovine allergens (a factor of 6.11) after adjusting for determinants milking by robot and surface size as square meters per cow. This means that using compost bedding in barns leads to more than six times higher personal exposure levels compared with saw dust bedding. Also rubberfilled mattress and rubber mats resulted in higher personal exposure levels than saw dust bedding. In personal 


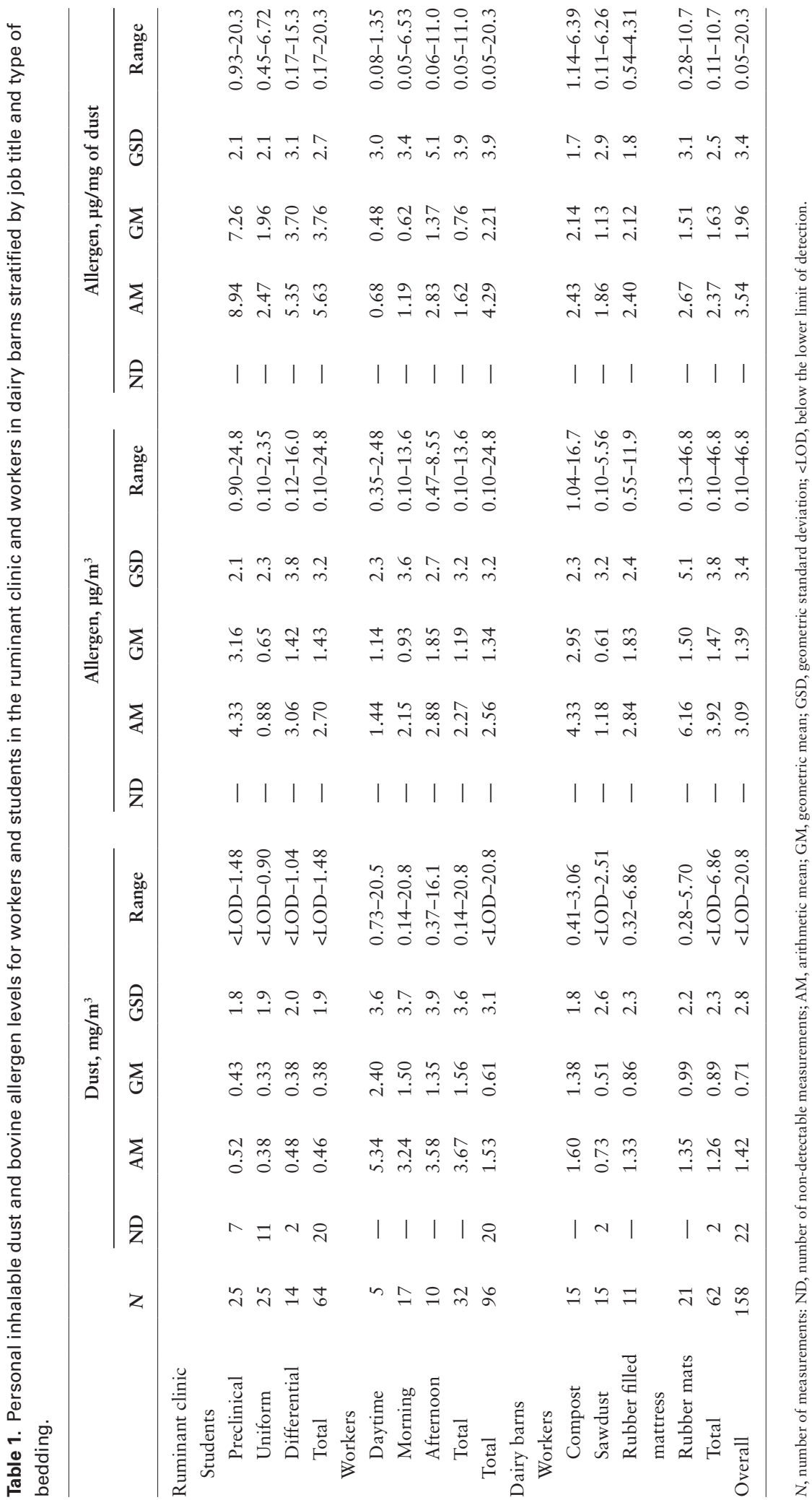




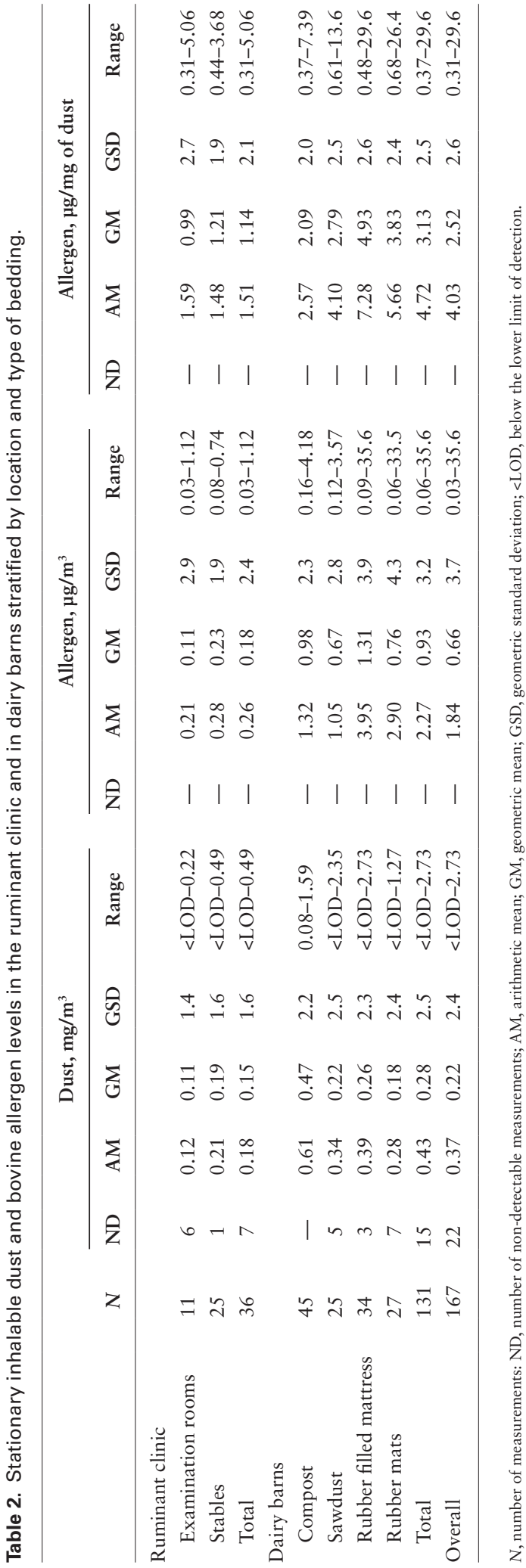

samples, milking by robot was significantly associated with lower bovine allergen levels (a factor of 0.35) compared with a traditional parlor. Similarly, increased surface size per cow was associated with slightly lower bovine allergen levels (a factor of 0.91). Looking at mixed-effect models for stationary samples, compost and rubber mats were as the strongest determinants of bovine allergens (a factor of 1.90). Like observed for personal samples, an increased surface size per cow was related to lower bovine allergen levels (a factor of 0.91), while milking by robot was not significantly associated to allergen levels. For personal allergen content no significant determinants were identified, which suggest that no major allergen content differences exist for investigated determinants.

Table 5 presents the estimated within- and betweenworker variance components. The between-worker variance, for exposure presented as $\mu \mathrm{g} / \mathrm{m}^{3}$, was moderately reduced (a reduction of $46 \%$ ) when all potential determinants were included as fixed effects simultaneously in the model. Milking by robot explained a considerable part of exposure variability (22\%), followed by bedding types explaining $18 \%$. The within-worker variance was not affected by determinants in the models. Betweenworker variances of exposure were lower for allergen content compared with variances in allergen concentrations. When all determinants were included simultaneously in the models, the reduction of between-worker variance was only $22 \%$.

\section{Discussion}

This study comprehensively quantified personal and stationary inhalable exposure levels of bovine allergens in a ruminant clinic as well as in several dairy barns. Additionally, airborne settled bovine allergen levels were measured using EDCs. In general, bovine allergens were measureable in all samples collected at different locations, despite levels of dust being non-detectable in some cases. All dust collection methods, including active and passive measurements, showed a wide range of bovine allergen levels. In the ruminant clinic, allergen levels largely differed based upon job titles and locations. Bedding appeared to be a significant determinant of bovine allergen exposure levels in dairy barns, especially compost bedding as the most influential determinant of exposure increase. Milking by robot as well as type of bedding were important exposure determinants explaining in part the variability in exposure.

Personal exposure levels to bovine allergens among workers measured in the ruminant clinic (GM $1.19 \mu \mathrm{g} / \mathrm{m}^{3}$ ) and dairy barns $\left(\mathrm{GM} 1.47 \mu \mathrm{g} / \mathrm{m}^{3}\right)$ were roughly two to 
Table 3. Airborne settled bovine allergen levels $\left(\mu \mathrm{g} / \mathrm{m}^{2}\right)$ in the ruminant clinic stratified by location.

\begin{tabular}{|c|c|c|c|c|c|c|}
\hline & $N$ & ND & $\mathrm{AM}$ & GM & GSD & Range \\
\hline Canteen & 2 & - & $5.40 \times 10$ & $5.40 \times 10$ & 1.0 & $5.35 \times 10^{4}$ to $5.44 \times 10$ \\
\hline Examination rooms & 3 & - & $5.70 \times 10^{2}$ & $5.68 \times 10^{2}$ & 1.1 & $5.09 \times 10^{5}$ to $6.45 \times 10^{2}$ \\
\hline Stables & 6 & - & $5.09 \times 10^{3}$ & $2.10 \times 10^{3}$ & 9.2 & $2.82 \times 10^{4}$ to $1.17 \times 10^{4}$ \\
\hline Total & 11 & - & $2.94 \times 10^{3}$ & $7.55 \times 10^{3}$ & 8.3 & $2.82 \times 10^{4}$ to $1.12 \times 10^{4}$ \\
\hline
\end{tabular}

$N$, number of measurements: ND, number of non-detectable measurements; AM, arithmetic mean; GM, geometric mean; GSD, geometric standard deviation.

Table 4. Parameter estimates in the final mixed effects model of the log-transformed personal and stationary exposure to bovine allergens in dairy barns in relation with exposure determinants.

\begin{tabular}{|c|c|c|c|c|c|c|c|c|c|}
\hline & \multirow[b]{2}{*}{ Determinant of exposure } & \multicolumn{4}{|c|}{ Allergen, $\mu \mathrm{g} / \mathrm{m}^{3}$} & \multicolumn{4}{|c|}{ Allergen, $\mu \mathrm{g} / \mathrm{mg}$ of dust } \\
\hline & & B & SE & $P$ value & FEC & B & SE & $P$ value & FEC \\
\hline \multirow[t]{8}{*}{ Personal samples } & Intercept $^{\mathrm{a}}$ & 8.46 & 0.76 & $<0.0001$ & - & - & - & - & - \\
\hline & Type of bedding & & & & & & & & \\
\hline & Compost & 1.81 & 0.57 & 0.003 & 6.11 & - & - & - & - \\
\hline & Rubber-filled mattress & 0.69 & 0.61 & 0.267 & 1.99 & - & - & - & - \\
\hline & Rubber mats & 0.66 & 0.53 & 0.223 & 1.93 & - & - & - & - \\
\hline & Sawdust & Ref & Ref & Ref & Ref & - & - & - & - \\
\hline & Milking by robot (Yes $v s$. no) & -1.06 & 0.45 & 0.024 & 0.35 & - & - & - & - \\
\hline & Surface size per cow & -0.10 & 0.05 & 0.035 & 0.91 & - & - & - & - \\
\hline \multirow[t]{8}{*}{ Stationary samples } & Intercept $^{\mathrm{a}}$ & 7.59 & 0.37 & 0.000 & - & 8.67 & 0.28 & 0.000 & - \\
\hline & Type of bedding & & & & & & & & \\
\hline & Compost & 0.64 & 0.30 & 0.038 & 1.90 & -0.45 & 0.23 & 0.048 & 0.64 \\
\hline & Rubber-filled mattress & -0.28 & 0.32 & 0.369 & 0.76 & -0.39 & 0.24 & 0.105 & 0.68 \\
\hline & Rubber mats & 0.66 & 0.29 & 0.027 & 1.96 & 0.30 & 0.22 & 0.172 & 1.35 \\
\hline & Sawdust & Ref & Ref & Ref & Ref & Ref & Ref & Ref & Ref \\
\hline & Milking by robot (Yes vs. no) & - & - & - & - & - & - & - & - \\
\hline & Surface size per cow & -0.10 & 0.03 & 0.002 & 0.90 & 0.05 & 0.02 & 0.043 & 1.05 \\
\hline
\end{tabular}

$\beta$, regression coefficient; SE, standard error; FEC, factor of exposure change.

${ }^{a}$ The intercept presents the exposure level for workers working in barns using sawdust after adjusting milking by robot and surface size per cow.

three times higher as the levels (mean $0.46 \mu \mathrm{g} / \mathrm{m}^{3}$ ) previously reported in Finish cowsheds (Virtanen et al., 1988a). Similarly, the stationary exposure levels of bovine allergens measured in dairy barns (GM $0.93 \mu \mathrm{g} / \mathrm{m}^{3}$ ) were also somewhat higher than the levels (means between 0.35 and $0.73 \mu \mathrm{g} / \mathrm{m}^{3}$ ) reported in the Finish study, while the observed levels in the cow barns within the ruminant clinic (GM $\left.0.23 \mu \mathrm{g} / \mathrm{m}^{3}\right)$ appeared to be relatively low (Virtanen et al., 1988a). Comparison of our results with Finish study needs to be made with care, as dust sampling methods are different (inhalable versus total dust fraction). More importantly, different allergen assays were applied, and a comparison between the assays is not available. Systematic differences between allergen assays have been reported before for other inhalable occupational allergens (Hollander et al., 1999; Bogdanovic et al., 2006).
Within the ruminant clinic, levels of bovine allergens for workers in the afternoon shift were notably higher than during the morning shift. Similar data are not available from other studies on bovine allergen exposures. Higher levels of bovine allergens during the afternoon shift contrast to previous findings on endotoxin exposure levels from the same samples (Samadi et al., 2011). This suggests that determinants of exposure for bovine allergens and endotoxin are not similar. This difference is probably to be a result of different tasks performed during work-shifts. Tasks performed by ruminant clinic workers in the afternoon shift were more observational resulting in walking close to cows, suggesting that more time spent in close contact to cows could be important determinants of cow allergen exposure.

Among students' job titles, highest levels of bovine allergens were observed in preclinical students. 


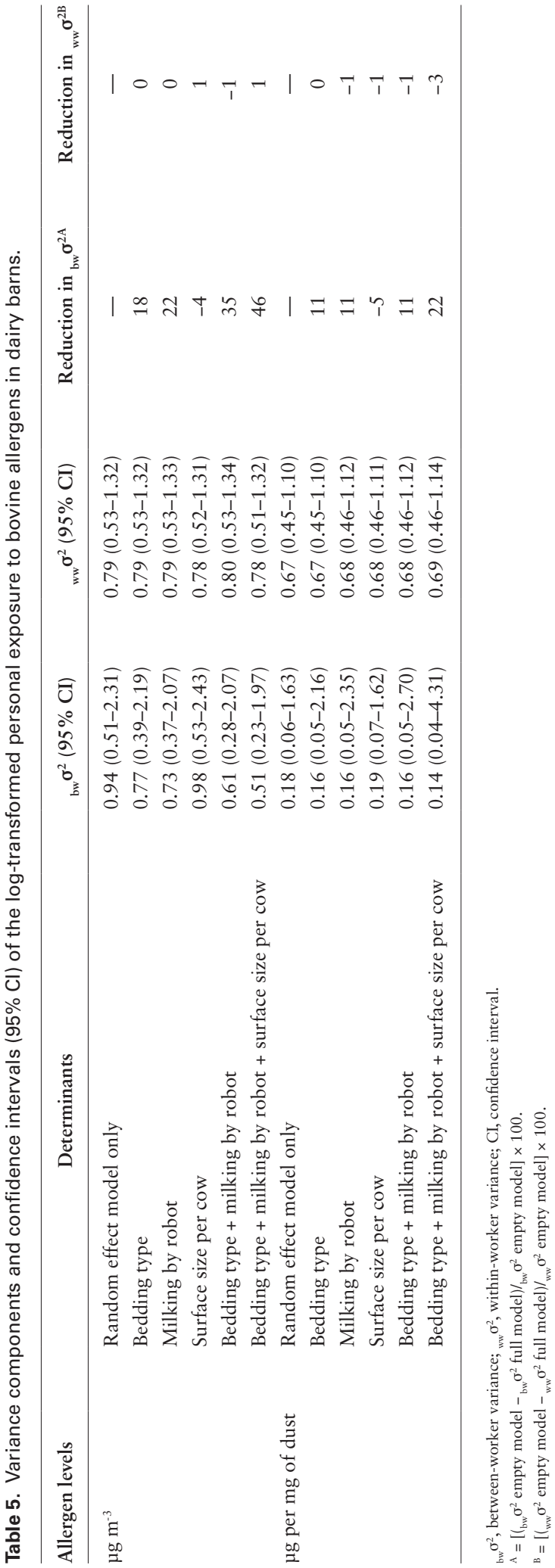

Surprisingly, exposure for this group of students was almost three times higher than exposure of workers within the ruminant clinic. Comparison with other studies is not possible. Nonetheless, preclinical students had relatively much more close contact to cows compared with the other students and also workers in the clinic, because preclinical students were measured during their practical clinically examining the cows. The doors of teaching rooms are often closed causing less ventilation. Furthermore, the sampling duration was much shorter for these preclinical students compared with the other students, resulting in only high-exposure task measurements. So all these likely lead to higher bovine allergen exposures. In the Netherlands generally only small number of farmers are working at the dairy farm, similar so, only small number of workers in the clinic are animal caretakers, further distinction in job titles within the farm workers and animal clinic workers is therefore not possible.

We found significant correlations between the levels of bovine allergens and inhalable dust levels, as previously observed in a companion animal setting for cat and dog allergens and inhalable dust (Samadi et al., 2010). The type of bedding material applied in dairy barns affected the levels of both personal and stationary bovine allergen exposures. These differences in levels due to bedding materials are comparable with those levels of laboratory animal allergens in relation to bedding materials applied in laboratory animal facilities (Kaliste et al., 2004). When looking at bovine allergen content based on bedding type, the patterns of allergen in $\mu \mathrm{g} / \mathrm{mg}$ of dust were different than observed for allergen levels as $\mu \mathrm{g} / \mathrm{m}^{3}$. The allergen content (in $\mu \mathrm{g} / \mathrm{mg}$ of dust) in personal samples for workers in dairy barns was in the same range for all bedding types. This suggests that bedding materials can influence the allergen exposure levels, possibly through dust formation including allergens, rather than through altered allergen content.

Slightly higher personal exposure levels of bovine allergens compared with those levels obtained by stationary measurements is in concordance with results of previous studies for other agents (Berger et al., 2005; Samadi et al., 2009, 2010, 2011, 2012b). This disparity can be elucidated by the fact that stationary measurements generally are performed at larger distances from the source. However, the results of stationary exposure monitoring for bovine allergens might be appropriate, for instance, to study effectiveness of measures diminishing exposure.

In this study, bovine allergen was measureable in passive airborne dust samples collected with EDCs within the ruminant clinic including cow stables, examination 
rooms and canteen. The allergen levels varied about 500-fold between different EDC samples collected in cow stables, which is in accordance with previous findings of wide range of bovine allergen levels obtained by EDCs in German and Danish farms (Zahradnik et al., 2011; Schlunssen et al., 2015). This wide range of EDC levels could be matched to similar wide ranges of bovine allergen levels observed in our personal and stationary measurements. The convenience of EDC settled airborne dust sampling, as an alternative technique versus personal and stationary exposure monitoring, is an apparent advantage for application at large scale. Exposure assessments in agricultural environmental settings such as animal farms are costly and time consuming since such settings spread over a large area and a limited number of workers within each farm are involved. So using EDC might be an appropriate method where the collection of personal or stationary exposure monitoring may be a problem. The detection of low levels of bovine allergens in the canteen are in accordance with the previously reported detection of low levels of cat and dog allergens in a similar setting; the canteen of a companion animal hospital (Samadi et al., 2010). This finding was parallel to results of two other studies, which reported detection of bovine allergens in dwellings of dairy farmers (Zahradnik et al., 2011; Schlunssen et al., 2015) and both studies proposed that bovine allergens are probably spread from the workplace by means of the clothes and hair of farmers. The presence of bovine allergens in canteen is most likely attributed to allergen transfer from the work environments through the clothes and hair of individuals. As yet, it is not known whether those low levels of exposure possess a health risk, as dose-response relationships for bovine allergen exposure and allergic outcomes are lacking. However, sensitization of family members to occupational allergens through household exposure has been reported (Liccardi et al., 2011).

Insight in exposure variation and their determinants is important. Between-worker variance in bovine allergen exposure was increased compared to the within-worker variance for measurements in dairy barns, indicating that between-worker variability in average exposure levels was higher than the day-to-day differences. The same holds for endotoxin and $\beta(1 \rightarrow 3)$-glucan exposures in the same dairy barns (Samadi et al., 2009). With only three determinants: bedding material used, cow surface area and presence/absence of a milking robot, $46 \%$ of total between-worker variability of bovine allergen exposures could be explained. The explained variance is considerably higher than the $12 \%$ explained variability reported by Schlünssen et al. in a Danish study using EDC samples despite inclusion of many other suspected determinants such as type of stable, animal density and ventilation (Schlunssen et al., 2015). However, this comparison should be made with caution since the Danish study included random samples of dairy barns while we included specific samples with known differences in bedding type and milking by robot. Milking by robot versus traditional parlor explained $22 \%$ of between-worker variability. We previously reported that milking by robot was a determinant of elevated dust exposure (Samadi et al., 2012b) as most likely workers spent more time on other dusty tasks. Paradoxically to dust, the levels of bovine allergens were negatively associated with the application of milking by robot, indicating that dust does only partly act as a substrate for bovine allergens perhaps due to less contact to cows. The increased levels of bovine allergens for bedding in personal and stationary models suggest the importance of bedding as a source. Given the personal samples were more affected than stationary samples suggest that other unexplored determinants such as type of tasks performed may be important as well. The within-worker variance could not be explained following inclusion of exposure determinants in the models, as the included determinants did not vary over time.

There is evidence that dairy farmers may get sensitized to bovine allergens (Rautalahti et al., 1987; Virtanen et al., 1988b; Ylonen et al., 1992; Doekes, 2000; Spiewak, 2001), suggesting that exposure to bovine allergens can be considered a risk factor for sensitization although conflicting results have been reported as well (Schlunssen et al., 2015). In general no doseresponse relationships are known. As the majority of dairy farmers often initiates working during childhood and usually continues working after 65 years of age, cumulative exposure to bovine allergen among dairy farmers could possibly be higher than the normal years of employment. The collected exposure data from this study could be a first step to explore the incidence/prevalence of sensitization and subsequent allergic airway diseases associated with the levels of bovine allergen exposure.

\section{Conclusion}

This study provides insights in exposure levels of bovine allergens using active and passive airborne dust measurements. The findings showed that workers and students in veterinary medicine were likely exposed to a wide range of bovine allergen levels. Exposure levels varied dependent on job titles, being highest among preclinical students who had the closest contact with the cows. Milking by robot or in a conventional parlor and type of 
bedding material appeared to be significant determinants of exposure in dairy barns. The exposure data collected in this study can be a first step to take investigation of exposure-response relations between bovine allergen exposure and development of bovine sensitization and/ or bovine allergen-induced asthma a step forward.

\section{Acknowledgement}

We would like to thank the workers of the ruminant clinic and the veterinary medicine students for their collaboration in exposure measurements. Giovanna Otten and Marijke Droppert are thanked for their help with exposure assessment measurements in dairy barns. Cooperating dairy farmers and the head of the ruminant clinic are thanked for willingness to cooperate in the study. No funding was provided for this research. The authors declare no conflict of interest relating to the material presented in this article.

\section{References}

Berger I, Schierl R, Ochmann U et al. (2005) Concentrations of dust, allergens and endotoxin in stables, living rooms and mattresses from cattle farmers in southern Bavaria. Ann Agric Environ Med; 12: 101-7.

Bogdanovic J, Wouters IM, Sander I et al. (2006) Airborne exposure to wheat allergens: optimised elution for airborne dust samples. J Environ Monit; 8: 1043-8.

Böhlandt A, Schierl R, Heizinger J et al. (2016) Cow hair allergen concentrations in dairy farms with automatic and conventional milking systems: from stable to bedroom. Int $J$ Hyg Environ Health; 219: 79-87.

Chapman MD, Wood RA. (2001) The role and remediation of animal allergens in allergic diseases. J Allergy Clin Immunol; 107(3 Suppl): S414-21.

Doekes G, Wouters I, de Vries J, (2000) IgE antibodies to cow allergens and respiratory health in dairy farmers in Denmark and The Netherlands. J Agric Saf Health; 5: 309-16.

Epstein S. (1948) Milker's eczema; an analysis of 42 cases. J Allergy; 19: 333-41.

Heutelbeck AR, Janicke N, Hilgers R et al. (2007) German cattle allergy study (CAS): public health relevance of cattle-allergic farmers. Int Arch Occup Environ Health; 81: 201-8.

Hollander A, Gordon S, Renström A et al. (1999) Comparison of methods to assess airborne rat and mouse allergen levels. I. Analysis of air samples. Allergy; 54: 142-9.

Kaliste E, Linnainmaa M, Meklin T et al. (2004) The bedding of laboratory animals as a source of airborne contaminants. Lab Anim; 38: 25-37.

Karjalainen A, Kurppa K, Virtanen S et al. (2000) Incidence of occupational asthma by occupation and industry in Finland. Am J Ind Med; 37: 451-8.

Liccardi G, D'Amato G, Antonicelli L et al.; Allergy Study Group of the Italian Society of Respiratory Medicine (SIMeR). (2011) Sensitization to horse allergens in Italy: a multicentre study in urban atopic subjects without occupational exposure. Int Arch Allergy Immunol; 155: 412-7.

Lubin JH, Colt JS, Camann D et al. (2004) Epidemiologic evaluation of measurement data in the presence of detection limits. Environ Health Perspect; 112: 1691-6.

Mäntyjärvi R, Parkkinen S, Rytkönen M et al. (1996) Complementary DNA cloning of the predominant allergen of bovine dander: a new member in the lipocalin family. $J$ Allergy Clin Immunol; 97: 1297-303.

Noss I, Wouters IM, Visser M et al. (2008) Evaluation of a lowcost electrostatic dust fall collector for indoor air endotoxin exposure assessment. Appl Environ Microbiol; 74: 5621-7.

Prahl P, Weeke B, Løwenstein H. (1978) Quantitative immunoelectrophoretic analysis of extract from cow hair and dander. Characterization of the antigens and identification of the allergens. Allergy; 33: 241-53.

Rautalahti M, Terho EO, Vohlonen I et al. (1987) Atopic sensitization of dairy farmers to work-related and common allergens. Eur J Respir Dis Suppl; 152: 155-64.

Rautiainen J, Rytkönen M, Virtanen T et al. (1997) BDA20, a major bovine dander allergen characterized at the sequence level, is Bos d 2. J Allergy Clin Immunol; 100: 251-2.

Samadi S, Heederik DJ, Krop EJ et al. (2010) Allergen and endotoxin exposure in a companion animal hospital. Occup Environ Med; 67: 486-92.

Samadi S, Rietbroek NN, Dwars RM et al. (2011) Endotoxin and $\beta-(1 \rightarrow 3)$-glucan exposure in poultry and ruminant clinics. J Environ Monit; 13: 3254-61.

Samadi S, Spithoven J, Jamshidifard AR et al. (2012a) Allergy among veterinary medicine students in The Netherlands. Occup Environ Med; 69: 48-55.

Samadi S, van Eerdenburg FJ, Jamshidifard AR et al. (2012b) The influence of bedding materials on bio-aerosol exposure in dairy barns. J Expo Sci Environ Epidemiol; 22: 361-8.

Samadi S, Wouters IM, Heederik DJ. (2013) A review of bioaerosol exposures and associated health effects in veterinary practice. Ann Agric Environ Med; 20: 206-21.

Samadi S, Wouters IM, Houben R et al. (2009) Exposure to inhalable dust, endotoxins, beta(1->3)-glucans, and airborne microorganisms in horse stables. Ann Occup Hyg; 53: 595-603.

Schlünssen V, Basinas I, Zahradnik E et al. (2015) Exposure levels, determinants and IgE mediated sensitization to bovine allergens among Danish farmers and non-farmers. Int J Hyg Environ Health; 218: 265-72.

Schram D, Doekes G, Boeve M et al.; PARSIFAL Study Group. (2005) Bacterial and fungal components in house dust of farm children, Rudolf Steiner school children and reference children-the PARSIFAL Study. Allergy; 60: 611-8.

Seward JP. (1999) Occupational allergy to animals. Occup Med; 14: 285-304.

Spiewak R. (2001) [Sensitization to cow and pig allergens among farmers in Eastern Poland]. Med Pr; 52: 351-4.

Terho EO, Husman K, Vohlonen I et al. (1985) Allergy to storage mites or cow dander as a cause of rhinitis among Finnish dairy farmers. Allergy; 40: 23-6. 
Virtanen T, Vilhunen P, Husman K et al. (1988a) Level of airborne bovine epithelial antigen in Finnish cowsheds. Int Arch Occup Environ Health; 60: 355-60.

Virtanen T, Vilhunen P, Husman K et al. (1988b) Sensitization of dairy farmers to bovine antigens and effects of exposure on specific IgG and IgE titers. Int Arch Allergy Appl Immunol; 87: 171-7.

Williams DL, Breysse PN, McCormack MC et al. (2011) Airborne cow allergen, ammonia and particulate matter at homes vary with distance to industrial scale dairy operations: an exposure assessment. Environ Health; 10: 72.

Williams DA, McCormack MC, Matsui EC et al. (2016) Cow allergen (Bos d2) and endotoxin concentrations are higher in the settled dust of homes proximate to industrial-scale dairy operations. J Expo Sci Environ Epidemiol; 26: 42-7.

Ylönen J, Mäntyjärvi R, Taivainen A et al. (1992) IgG and IgE antibody responses to cow dander and urine in farmers with cow-induced asthma. Clin Exp Allergy; 22: 83-90.

Ylönen J, Virtanen T, Rytkönen M et al. (1994) Quantification of a major bovine allergen by a two-site immunometric assay based on monoclonal antibodies. Allergy; 49: 707-12.

Zahradnik E, Sander I, Bruckmaier L et al. (2011) Development of a sandwich ELISA to measure exposure to occupational cow hair allergens. Int Arch Allergy Immunol; 155: 225-33. 